\title{
Caracterização de compósitos de Poliestireno de Alto Impacto (HIPS) reforçados com fibras de coco verde para eventual aplicação na indústria automobilística
}

\author{
Characterization of High Impact Polystyrene (HIPS) composites \\ reinforced with green coconut fibers for eventual application in the \\ automobile industry
}

\author{
1 Gilmara Brandão Pereira brandaoconsultoria.treinamento@gmail.com \\ 1 Glayce Cassaro Pereira \\ 2 Kelly Cristina Coelho de Carvalho Benini \\ 1 Cirlene Fourquet Bandeira \\ 3 Sérgio Roberto Montoro \\ UniFOA/MEMAT \\ 2 UNESP/FEG \\ 3 UniFOA/MEMAT, FATEC Pindamonhangaba
}

\section{Resumo}

Estudos estão sendo realizados para geração de produtos que reduzem o impacto ambiental. Dentre esses estudos, a obtenção de compósitos poliméricos reforçados com fibras naturais vem se destacando. Um exemplo desse tipo de compósito é o reforçado com fibras de coco verde, uma vez que a geração de resíduos provenientes do coco vem aumentando no Brasil devido à industrialização da água de coco. Esse trabalho teve como objetivos processar compósitos de poliestireno de alto impacto (HIPS), reforçados com fibras de coco verde e verificar sua possível aplicação na indústria automobilística. Foram preparadas amostras de compósito nas proporções 10, 20 e $30 \%$ em massa de fibra. Foram realizadas análises para determinação da massa específica, dureza Shore A e resistência ao impacto. Os resultados de massa específica e da dureza Shore A não sofreram alterações, devido à inserção das fibras de coco no HIPS. Entretanto, foi observado que a resistência ao impacto sofreu redução, devido à inserção das fibras. Cabe ressaltar que a diminuição da resistência ao impacto não inviabiliza a utilização do compósito HIPS/fibra de coco, uma vez que somente demonstra que esse material pode ser empregado em peças que não são submetidos a grandes esforços mecânicos, tais como as que compõem o interior de automóveis.

\section{Palavras-chave}

Compósitos; dureza Shore A; massa específica; resistência ao impacto, fibra de coco verde; HIPS.

\begin{abstract}
Studies are being carried out to generate products that reduce environmental impact. Among these studies, the obtaining of polymeric composites reinforced with natural fibers has been highlighting. An example of this type of composite is the reinforcement with green coconut fibers, since the generation of residues from the coconut is increasing due mainly to the industrialization of coconut water in Brazil. This work aims to process composite high impact polystyrene (HIPS) reinforced with coconut fiber and verify their possible application in the automotive industry. For this HIPS composite reinforced samples were prepared with coconut fibers in the proportions 10, 20 and $30 \%$ by weight of fiber. Analyses were performed to determine the density, Shore $A$ hardness and impact resistance in processed composites. It was found that the results of density and Shore $A$ hardness did not change due to the inclusion of coconut fibers in the HIPS. However, it was observed that the impact resistance was reduced due to the insertion of the fibers. It is noteworthy that the decrease in impact resistance does not preclude the use of composite HIPS / coconut fiber, since only demonstrates that this material can be used on parts that are not subjected to large mechanical stresses such as those that make up the interior automobiles.
\end{abstract}

\section{Keywords}

Composites, Shore A hardness; Especific mass; Impact Resistance; coconut fiber; HIPS.

\section{Como você deve citar?}

PEREIRA, Gilmara Brandão et al. Caracterização de compósitos de Poliestireno de Alto Impacto (HIPS), reforçados com fibras de coco verde para eventual aplicação na indústria automobilística. Cadernos UniFOA, Volta Redonda, n. 34, p. 15-22, ago. 2017. 
Caracterização de compósitos de Poliestireno de Alto Impacto (HIPS)

reforçados com fibras de coco verde para eventual aplicação na indústria automobilística

\section{INTRODUÇÃO}

Nos últimos anos, vem crescendo o interesse pelo desenvolvimento de novas tecnologias que propiciem utilização de produtos com menor grau de impacto ambiental. Sendo assim, várias pesquisas estão sendo realizadas na área de compósitos poliméricos, no intuito de garantir a preservação ambiental. As pesquisas que mais se destacam são aquelas relacionadas à utilização de fibras naturais (MARINELLI et al., 2008; SILVA et al., 2009).

A partir do surgimento de políticas ambientais, surgiu o conceito de desenvolvimento sustentável, que, na indústria automobilística, vem associando à gestão socioambiental, à qualidade e à competitividade. É sabido que o futuro do automóvel e de sua indústria passará pela capacidade das indústrias de reduzir e/ou compensar seus efeitos danosos sobre o meio ambiente, desde a produção de materiais à reciclagem de autopeças e de veículos em seu fim de vida (MULINARI, 2009).

Em busca de alternativas para a substituição e/ou redução dos insumos de fontes não renováveis, a indústria automobilística tem desenvolvido pesquisas sobre a utilização das fibras naturais. Tais fibras já são utilizadas há um tempo na fabricação de peças de automóveis que, dentre suas formas de aplicação, pode-se citar o enchimento de bancos e encostos de cabeça, as laterais e os painéis de portas, o painel de instrumentos, canais de ar, revestimentos do teto, caixa de rodas, dentre outros. Para tais aplicações, são utilizadas fibras naturais, como, por exemplo, o bagaço de cana, sisal, juta, curauá, fibra de coco, entre outras (MULINARI et al., 2010; CARVALHO et al., 2010; OLIVEIRA, 2009; IBRAHIM et al., 2010).

Em se tratando especificamente da fibra de coco, de 1990 a 2009, o Brasil passou de $10^{\circ}$ para $4^{\circ}$ maior produtor mundial de coco, sendo que existem perspectivas de crescimento não só na produtividade, como também na abrangência do mercado (MARTINS et al., 2013).

Um dos motivadores foi a industrialização da água de coco no Brasil. Isso fez com que houvesse um aumento no consumo de coco verde e, consequentemente, uma redução da vida útil dos aterros, em virtude do aumento da geração de resíduos provenientes da casca do coco, representando um risco ao meio ambiente (BARBOSA et al., 2010).

A utilização de fibra de coco verde na produção de compósitos é de suma importância, pois não só reduz a geração de resíduos como também é um processo barato, natural e renovável, o que aproxima ainda mais o estudo em questão do conceito de sustentabilidade (BARBOSA et al., 2010).

Dentre as matrizes dos materiais compósitos, a mais utilizada é a matriz polimérica em virtude de versatilidade de formulação e baixo custo de processamento (CALLISTER, 2002).

Muitos polímeros demonstram comportamento frágil à fratura (ROVERE et al., 2008). 0 poliestireno de alto impacto (PSAI) ou HIPS (High Impact Polystyrene), por sua vez, tem aumentado sua resistência à fratura e tenacidade em função de uma segunda fase de polibutadieno (PB). O ponto de amolecimento do HIPS, entre 87 e $100^{\circ} \mathrm{C}$, e solubilidade parcial em hidrocarbonetos e cetonas, bem como sua temperatura de processamento abaixo da temperatura de degradação das fibras naturais e ainda seu fácil processamento, faz dele uma alternativa viável de matriz para compósitos reforçados com fibras naturais (LEVY NETO, 2006).

Como parcialmente citado anteriormente, a escolha do HIPS para esse estudo se deu ao seu baixo custo, quando comparado a outros termoplásticos, além do HIPS apresentar algumas vantagens, como 
rigidez adequada, facilidade de processamento e por poder ser processado em temperaturas abaixo da temperatura de degradação da celulose.

Dentro desse contexto, o presente trabalho visou à caracterização de compósitos reforçados com fibras da casca do coco verde em matriz de poliestireno de alto impacto (HIPS), tendo como objetivo uma possível substituição dos materiais utilizados atualmente para a confecção de peças utilizadas nos automóveis.

\section{MATERIAIS E MÉTODOS}

\subsection{Compósitos}

Todos os compósitos que foram caracterizados nesse trabalho foram cedidos pela UNESP/FEG, campus de Guaratinguetá/SP. Os compósitos foram processados usando-se o HIPS 825 e reforçados com fibras de coco verde nas frações volumétricas de $10 \%, 20 \%$ e $30 \%$, em massa.

\subsection{Determinação da massa específica real via picnometria de hélio}

Utilizou-se a técnica de picnometria de hélio para determinação da massa específica real do HIPS puro e das amostras dos compósitos nas diferentes proporções. Foi utilizado um analisador de densidade real, marca Quantachrome Instruments, modelo Ultrafoam 1200e, locado no Laboratório de Caracterização de Compósitos do DMT/UNESP/FEG. A temperatura do ensaio foi de $20^{\circ} \mathrm{C}$ e os resultados foram expressos a partir da média aritmética de três leituras de uma mesma amostra de cada família $(10 \%, 20 \%$ e $30 \%)$.

\subsection{Determinação da dureza Shore A}

Para obtenção da dureza Shore A do HIPS puro e dos compósitos, foi utilizado um durômetro portátil, marca CV Instrument Limited, modelo CV Shore Durometers - Analogue, locado no Laboratório de Metrologia da empresa Maxion Structural Components - Unidade de Cruzeiro/SP. A temperatura do ensaio foi de $20^{\circ} \mathrm{C}$ e resultados foram expressos a partir da média aritmética de três leituras de uma mesma amostra de cada família ( $10 \%, 20 \%$ e $30 \%)$.

\subsection{Determinação da resistência ao impacto}

Para a determinação da resistência ao impacto do HIPS puro e dos compósitos, foi utilizada uma máquina de impacto Canti Lever XJU-22 do tipo Izod, provida de martelo de 5,5 J, locada no Laboratório de Ensaios Mecânicos da Universidade de São Paulo (USP/EEL/Área 2). Foram utilizados corpos-de-prova com dimensões $12 \times 55 \times 3 \mathrm{~mm}$ e os resultados foram expressos a partir da média aritmética dos cinco corpos-de-prova ensaiados de cada família (10\%, $20 \%$ e $30 \%)$.

\section{RESULTADOS E DISCUSSÃO}

\subsection{Massa específica}

A partir das análises de picnometria de hélio, verificou-se que a adição da fibra de coco ao HIPS não acarretou um aumento na massa específica dos compósitos processados, quando comparado ao HIPS puro, conforme apresentado na Tabela 1. Esses resultados se mostraram interessantes, pois futu- 
Caracterização de compósitos de Poliestireno de Alto Impacto (HIPS)

reforçados com fibras de coco verde para eventual aplicação na indústria automobilística

ras peças produzidas com os compósitos reforçados com as fibras de coco apresentarão praticamente o mesmo peso, se elas fossem produzidas com HIPS puro. Entretanto, a utilização de, por exemplo, $30 \%$ de fibras de coco $\left(\rho=1,1151 \mathrm{~g} / \mathrm{cm}^{3}\right)$, acarretará uma redução de $70 \%$ no consumo de HIPS puro $\left(\rho=1,1064 \mathrm{~g} / \mathrm{cm}^{3}\right)$ e, consequentemente, uma redução no custo das peças produzidas.

Tabela 1 - Resultados das massas específicas por meio da análise de picnometria de hélio.

\begin{tabular}{cc}
\hline AMOSTRAS & Massa Específica $\left(\mathbf{g} / \mathbf{c m}^{3}\right)$ \\
\hline HIPS PURO & $1,1064 \pm 0,0074$ \\
Fibra de Coco & $1,5844 \pm 0,0098$ \\
CT-10 & $1,1281 \pm 0,0123$ \\
CT-20 & $1,1589 \pm 0,0018$ \\
CT-30 & $1,1151 \pm 0,0032$ \\
\hline
\end{tabular}

Fonte: dos autores, 2017.

Em seu estudo, Pereira (2016) constatou que a adição da fibra de bagaço de cana-de-açúcar ao HIPS também não acarretou um aumento na massa específica dos compósitos processados, quando comparado ao HIPS puro.

\subsection{Dureza Shore A}

Os resultados encontrados por meio da análise de dureza demonstram que o HIPS não sofreu alterações nessa propriedade, quando acrescidos de fibra de coco. Os compósitos demonstraram, assim como o HIPS puro, resultados que podem classificar o HIPS, tanto os compósitos, como materiais muito duros, pois apresentaram valores de dureza na faixa entre 90-100 Shore A (FINOCCHIO, 2016). A Tabela 2 apresenta os resultados de dureza Shore A do HIPS puro e dos compósitos de fibra de coco verde. Considerando uma possível aplicação na indústria automobilística, os resultados de dureza foram satisfatórios, pois a inserção das fibras de coco verde não alterou, de modo significativo, a dureza Shore A do HIPS.

Tabela 2 - Resultados de dureza Shore A do HIPS puro e dos compósitos HIPS/fibra de coco verde.

\begin{tabular}{cc}
\hline AMOSTRAS & DUREZA SHORE A \\
\hline HIPS PURO & $97,7 \pm 0,8$ \\
CT-10 & $97,7 \pm 0,6$ \\
CT-20 & $97,0 \pm 1,0$ \\
CT-30 & $98,0 \pm 1,0$ \\
\hline
\end{tabular}

Fonte: dos autores, 2017.

Os resultados de dureza demostraram que ocorreu uma boa interação fibra/matriz nos compósitos analisados. No trabalho realizado por De Paula (2014), o autor desenvolveu compósitos poliméricos reforçados com pallets industriais, usando o Polipropileno (PP) como matriz e o PP-g-MAH como agente compatibilizante. Os resultados obtidos revelaram que a adição das fibras à matriz de PP proporcionou aumento na dureza, quando comparado ao polímero puro.

No trabalho realizado por PEREIRA et al., (2016), os resultados da dureza Shore A indicaram que a adição da fibra de bagaço de cana-de-açúcar ao HIPS não acarretou alterações nos valores da dureza nos compósitos, quando comparados ao HIPS puro. 


\subsection{Resistência ao impacto}

A partir das análises de resistência ao impacto, verificou-se que a adição da fibra de coco ao HIPS acarretou uma diminuição na resistência ao impacto, quando comparados ao HIPS puro. A Tabela 3 apresenta os resultados dos ensaios de impacto realizados no HIPS puro e nos compósitos CT-10, CT-20 e CT-30.

A diminuição da resistência ao impacto pode ter ocorrido, devido ao fato de que o reforço promoveu uma redução da ductilidade dos compósitos. Assim, o caráter mais frágil dos compósitos foi maior, de acordo com o aumento do volume de fibra, lembrando que a ductilidade corresponde à elongação total material, devido à deformação plástica.

Tabela 3 - Resultados da resistência ao impacto do HIPS puro e dos compósitos HIPS/fibra de coco verde.

\begin{tabular}{ccc}
\hline AMOSTRAS & Energia Absorvida $(\mathrm{J})$ & Resistência ao Impacto $\left(\mathbf{k J} / \mathbf{m}^{2}\right)$ \\
\hline HIPS PURO & $1,887 \pm 0,424$ & $57,095 \pm 12,917$ \\
CT-10 & $0,726 \pm 0,105$ & $22,076 \pm 3,198$ \\
CT-20 & $0,705 \pm 0,067$ & $21,456 \pm 2,032$ \\
CT-30 & $0,636 \pm 0,095$ & $19,350 \pm 2,907$ \\
\hline
\end{tabular}

Fonte: dos autores, 2017

No trabalho realizado por Benini (2011), constatou-se, através de ensaios de tração, que a adição de fibras de coco na matriz polimérica de HIPS praticamente não alterou a deformação do material até a tensão máxima (parcela referente à deformação elástica), porém provocou uma redução em torno de $85 \%$ na deformação total do material, conforme apresentado na Figura 1.

Figura 1 - Gráfico Tensão x Deformação para o HIPS e para os compósitos HIPS/fibra de coco verde (BENINI, 2011).

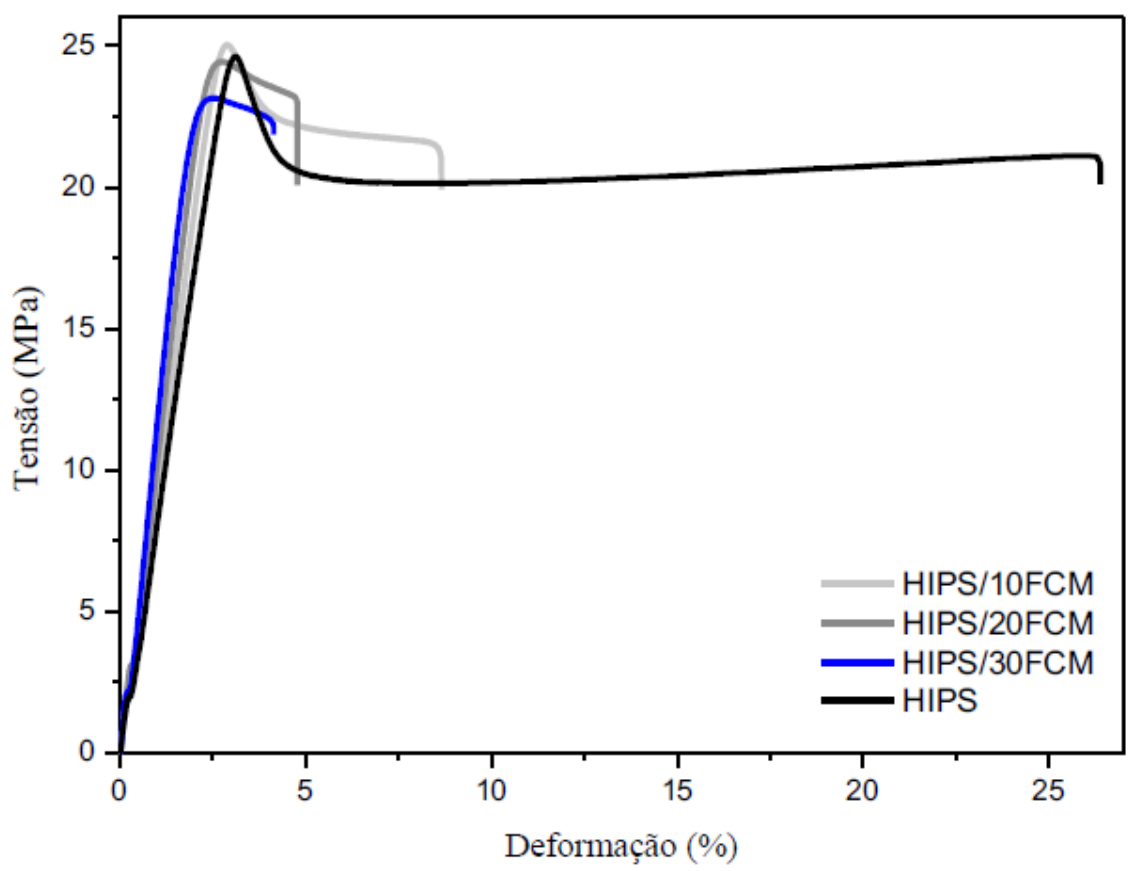

Fonte: BENINI, 2011. 
Ainda segundo Benini (2011), com o aumento do volume de fibras, foi possível observar uma redução na elongação total dos compósitos, conforme apresentado na Tabela 4.

Tabela 4 - Alongamento total do HIPS puro e dos compósitos com fibras de coco, obtido no trabalho realizado por BENINI (2011) [9].

\begin{tabular}{cc}
\hline AMOSTRAS & ALONGAMENTO TOTAL (\%) \\
\hline HIPS PURO & $26,2 \pm 8,1$ \\
CT-10 & $9,4 \pm 0,7$ \\
CT-20 & $5,0 \pm 0,3$ \\
CT-30 & $3,8 \pm 0,2$ \\
\hline
\end{tabular}

Fonte: BENINI, 2011.

Esse fenômeno pode ser associado ao fato de que o reforço promoveu uma redução da ductilidade do material e, consequentemente, acarretou a redução da resistência ao impacto, como mostrado na Tabela 3 (BENINI, 2011).

Pode-se justificar a redução da resistência ao impacto também pela diminuição da tenacidade dos compósitos com o aumento da adição de fibras, lembrando que a ela corresponde à capacidade do material de absorver energia até sua ruptura e que pode ser representada pela área da curva tensão versus deformação, conforme mostrado na Figura 2, que, por sua vez, evidencia claramente a resistência ao impacto dos materiais, mostrando a influência da inserção de fibras de coco na resistência do HIPS.

Figura 2 - Resistência ao impacto do HIPS puro e dos compósitos HIPS/fibra de coco verde.

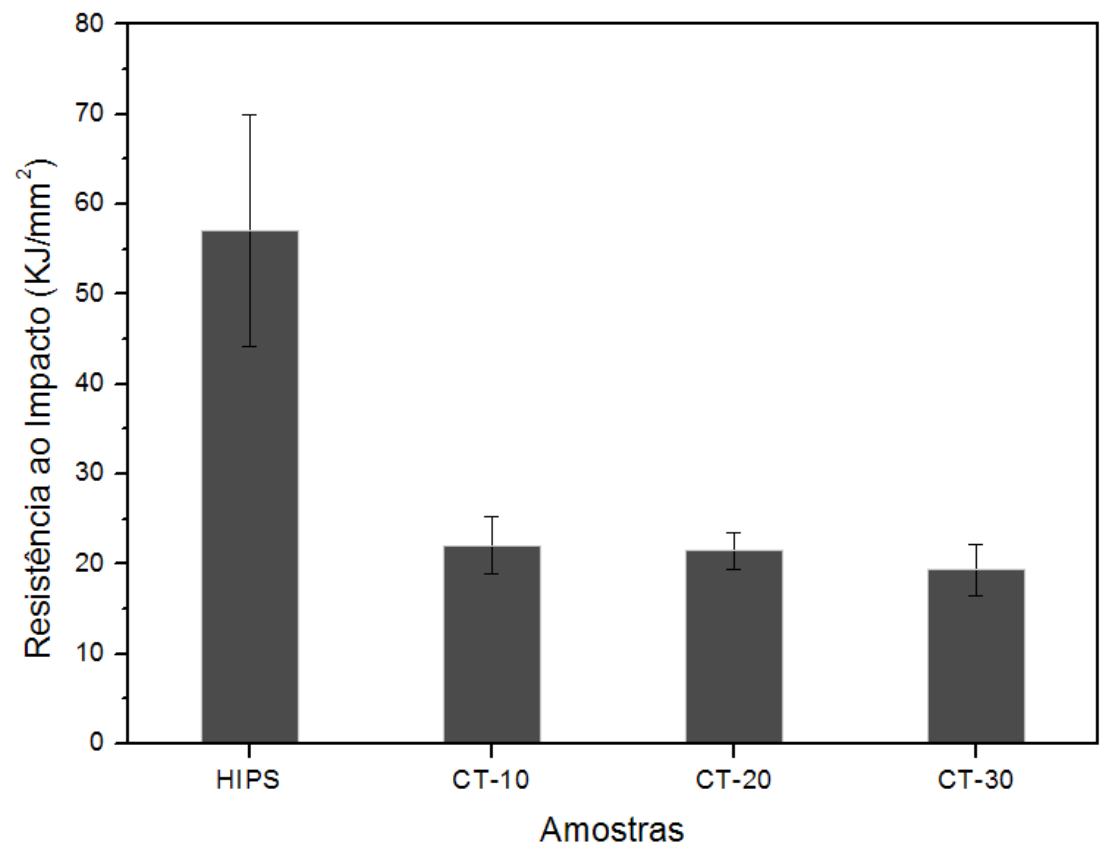

Fonte: dos autores, 2017.

No trabalho realizado por Pereira et al., (2016), os autores verificaram também que a adição da fibra de bagaço de cana-de-açúcar ao HIPS acarretou uma diminuição na resistência ao impacto, quando comparados ao HIPS puro. Os autores também justificaram a redução da resistência ao impacto pela diminuição da tenacidade dos compósitos com o aumento da adição de fibras. 
Já no trabalho realizado por Souza et al., (2012) os autores concluíram que a inserção de fibras provenientes do resíduo do suco do abacaxi na matriz de polipropileno influenciou positivamente na resistência ao impacto, quando comparado ao polipropileno puro. No entanto, observaram que, ao inserir maior quantidade $(20 \% \mathrm{~m} / \mathrm{m})$ de fibras na matriz polimérica, a energia dissipada aumentou, quando comparada ao compósito com menor fração volumétrica.

\section{CONCLUSÕES}

Com a análise dos resultados obtidos neste trabalho, foi possível avaliar o efeito da adição de fibras de coco verde no HIPS sobre algumas propriedades dos compósitos de HIPS reforçados com essas fibras e sua possível aplicação na indústria automobilística.

A partir das análises de picnometria de hélio, verificou-se que a adição da fibra de coco ao HIPS não acarretou um aumento na massa específica dos compósitos processados, quando comparado ao HIPS puro.

Os resultados de dureza Shore A indicaram que a adição da fibra de coco ao HIPS não acarretou alterações nos valores da dureza nos compósitos, quando comparados ao HIPS puro.

Foi possível constatar que a adição da fibra de coco ao HIPS acarretou uma diminuição na resistência ao impacto, quando comparados ao HIPS puro. Portanto, com a adição de fibras de coco verde no HIPS, obteve-se um material com até $30 \%$ menos polímero e com propriedades mecânicas viáveis para determinadas aplicações do HIPS, em que o custo e a dureza Shore A são mais importantes que a resistência ao impacto.

\section{REFERÊNCIAS}

BARBOSA JR, V.; RAMIRES, E. C.; RAZERA, I. A. T.; FROLLINI, E. Biobased composites from tanninphenolic polymers reinforced with coir fibers. Industrial Crops and Products, v.32, n.3, p.305-312, 2010.

BENINI, K. C. C. C. Desenvolvimento e caracterização de compósitos poliméricos reforçados com fibras lignocelulósicas: HIPS/fibra da casca do coco verde e bagaço de cana de açúcar. 2011. Dissertação (Mestrado em Engenharia Mecânica). Faculdade de Engenharia de Guaratinguetá - Universidade Estadual Paulista, Guaratinguetá, 2011.

CALLISTER Jr. W. D. Ciência e engenharia de materiais: uma introdução. 5. ed. Rio de Janeiro, R: LTC, 2002.

CARVALHO, K.C.C.; MULINARI, D.R.; VOORWALD, H.J.C.; CIOFFI, M.O.H. Chemical modification effect on the mechanical properties of HIPS/ coconut fiber composites. BioResources, v.5, n.2, p.1143-1155, 2010.

DE PAULA, G. H. R. Desenvolvimento de compósitos poliméricos reforçados com pallets industriais. Monografia (Design). Centro Universitário de Volta Redonda, Volta Redonda, 2014.

FINOCCHIO, M. A. F. Notas de aulas. Disponível em: <http://paginapessoal.utfpr.edu.br/mafinocchio/ disciplinas-da-graduacao/et35p-materiais-e-equipamento-eletricos/DUREZA.pdf $>$. Acesso em: 10 maio 2016. 
Caracterização de compósitos de Poliestireno de Alto Impacto (HIPS)

reforçados com fibras de coco verde para eventual aplicação na indústria automobilística

IBRAHIM, M. M.; DUFRESNE, A.; EL-ZAWAWY, W. K.; AGBLEVOR, F. A. Banana fibers and microfibrils as lignocellulosic reinforcements in Polymer composites. Carbohydrate Polymers, v.81, p.811-819, 2010.

LEVY NETO, F., PARDINI, L. C. Compósitos estruturais: ciência e tecnologia. São Paulo: Edgard Blucher Editora, 2006.

MARINELLI, A. L.; MONTEIRO, M. R.; AMBRÓSIO, J. D.; BRANCIFORTI, M. C.; KOBAYASHI, M.; NOBRE, A. D. Desenvolvimento de compósitos poliméricos com fibras vegetais naturais da biodiversidade. Polímeros: Ciência e Tecnologia, vol. 18, nº 2, p. 92-99, 2008.

MARTINS, A.; TOSHIKO, W.; SILVA, P.; BORELLI, C.; MARCICANO, J. SANCHES, R. Aproveitamento de fibra de coco verde para aplicabilidade têxtil. REDIGE v. 4, n. 02, ago. 2013.

MULINARI, D. R. Comportamento térmico, mecânico e morfológico dos compósitos de polietileno de alta densidade reforçados com fibras de celulose do bagaço de cana de açúcar. 2009. Tese (Doutorado em Engenharia Mecânica). Faculdade de Engenharia de Guaratinguetá - Universidade Estadual Paulista, Guaratinguetá, 2009.

MULINARI, D. R.; VOORWALD, H. J. C.; CIOFFI, M. O. H.; DA SILVA, M. L. C. P.; ROCHA, G. J. M. Surface modification of sugarcane bagasse cellulose and its effect on mechanical and absorption properties of sugarcane bagasse cellulose/HDPE composites. Bioresource, v.5, p.661-671, 2010.

OLIVEIRA, A. L. C. O. Modificação da fibra de linho por esterificação através de sistemas anidridos. 2009. Monografia (Engenharia de Materiais) - Faculdade de Engenharia de Guaratinguetá - Universidade Estadual Paulista, Guaratinguetá, 2009.

PEREIRA, G. C. Caracterização de compósitos HIPS/bagaço de cana e confecção de manual operacional para aplicação na indústria de Pet Shop. 2016. Dissertação (Mestrado Profissional em Materiais). Centro Universitário de Volta Redonda, Volta Redonda, 2016.

PEREIRA, G. C.; PEREIRA, G. B.; BANDEIRA, C. F.; MONTORO, S. R. Influência da quantidade de fibras na dureza e resistência ao impacto de compósitos HIPS/bagaço de cana para aplicação na indústria de pet shop. Em: XX Encontro Latino Americano de Iniciação Científica, XVI Encontro Latino Americano de Pós-Graduação e VI Encontro de Iniciação à Docência - Universidade do Vale do Paraíba, São José dos Campos, 2016.

ROVERE, J.; CORRÊA. C. A.; GRASSI, V. G.; PIZZOL, M. F. Caracterização morfológica do poliestireno de alto impacto. Polímeros: Ciência e Tecnologia, vol. 18, $n^{\circ}$ 1, p. 12-19, 2008.

SILVA, R.; HARAGUCHI, S. K.; MUNIZ, E. C.; RUBIRA, A. F. Aplicações de fibras lignocelulósicas na química de polímeros e em compósitos. Quim. Nova, Vol. 32, No. 3, 661-671, 2009.

SOUZA, T. A.; ROCHA, G. J. M.; MULINARI, D. R. Avaliação da resistência ao impacto de compósitos reforçados com fibras naturais. Cadernos UniFOA, Volta Redonda, n. 19, p. 21-29, 2012. 